\title{
Biomechanical analysis of conventional and locking compression plate (LCP) for treating fibula fracture: A finite element study
}

\author{
Mohamad Hafiz Mohamad Afandi a, Muhammad Hanif Ramlee ${ }^{a, *}$, Gan Hong Seng ${ }^{b}$, Mohammed \\ Rafiq Abdul Kadir a,c \\ a Medical Devices and Technology Centre (MEDITEC), Institute of Human Centered Engineering (i-Humen), School of Biomedical Engineering and \\ Health Science, Faculty of Engineering, Universiti Teknologi Malaysia, Skudai 81310, Johor, Malaysia \\ b Universiti Kuala Lumpur, British Malaysian Institute, Batu 8 Jalan Sg. Pusu, 53100 Gombak, Selangor, Malaysia \\ c Sports Innovation and Technology Centre (SITC), Institute of Human Centered Engineering (iHumen), Universiti Teknologi Malaysia, Skudai \\ 81310, Johor, Malaysia
}

*Corresponding author: muhammad.hanif.ramlee@biomedical.utm.my

\section{Article history}

Received 1 August 2019

Revised 12 February 2020

Accepted 1 April 2020

Published Online 17 August 2020

\begin{abstract}
1) Background: Due to questionable effectiveness of malleolar fracture fixation, biomechanical study was conducted to compare the stability of One Third Tubular (OTT) Plate and Locking Compression Plate (LCP); 2) Methods: CT image of bone was used to develop 3D bone model while the plate was constructed in Solidwork with varied number of screws. Further, finite element study was conducted for both models where the bone and plate were defined as homogenous and isotropic material properties; 3) Results: For LCP, the highest VMS observed at the plate for 3 screws was $484 \mathrm{MPa}$, whereas for 5 screws plate was $667 \mathrm{MPa}$. Meanwhile, for OTT, the highest VMS at plate was observed for 3 screws was $300.5 \mathrm{MPa}$, whereas for 5 screw plate was $127.5 \mathrm{MPa}$. 4) Conclusion: Based on the results, it can be noted that the usage of 3 screw causing a lower VMS at plate compared to 5 screws. However, the relation is only valid for LCP. On the other hand, for OTT, 5-screw constructs giving a low VMS than 3-screw constructs. It can be concluded that the optimum stabilities of OTT and LCP were found at 5 screws and 3 screws, respectively.
\end{abstract}

Keywords: One third tubular plate; locking compression plate; finite element analysis; fibula

\section{INTRODUCTION}

From 2007 to 2011, a hospital from the United States of America (USA) reported that the ankle has the highest number of fractures at lower limb which was 280,933 cases. This shows that malleolar fracture has the highest ranking of occurrence specifically at the ankle joint (Shibuya et al., 2007). In order to treat malleolar fracture, a surgery should be conducted to restore the fracture into the original formation of the structure. One of the methods in restoring the ankle fracture is the open reduction and internal fixation (ORIF) method. The ORIF method is known as an open surgery to fix fracture bone that normally happened in a worst-case scenario where the fractures are displaced, not stable, and has a high rate of complications. Then, internal fixation is used to fix the fracture bone until healing process takes place. Until now, there is no problem with the conventional plate, which is one third tubular plate (OTT) if the patient's bone density is in normal condition. Another design that have been used to restore the ankle fracture is the locking compression plate (LCP). Noted that the use of these two plates is favourable in treating ankle fracture specifically at fibula region (Labronici et al., 2016).

In clinical practises, the effect of complication from the application of plate fixation on the fibula fracture is $6.2 \%$ for the LCP fixation and $1.4 \%$ for the OTT plate fixation (Labronici et al., 2016). These cases will require medical surgeons to conduct second surgery for removing the implant. The complications that reported in the literature are from various aspects such as implant loosening, infection, mal-union, nonunion, and misalignment. As far as the authors are concerned, there is no study performed to evaluate the biomechanical properties of LCP and OTT in treating fibula fracture. Therefore, this study was conducted to compare the stability and stress distribution of LCP and OTT for better understanding on biomechanics principle of these two designs.

\section{METHODOLOGY}

\section{Bone reconstruction and implant design}

Computed Tomography (CT) images dataset was used to reconstruct ankle bone model, consisting of four bones which are fibula, tibia, talus, and calcaneus. The bone images provided by CT Scan were used to develop three-dimensional (3D) model of bone via Mimic software (10.01, Materialise, Belgium). Next, the 3D images undergone a process call meshing where the tetrahedral mesh size was $3.0 \mathrm{~mm}$ (Raja Izaham et al., 2012). An opening of $2 \mathrm{~mm}$ gap was simulated by removing a cylindrical-shaped bone from the distal part of the fibula.

The Conventional plate and Locking Compression plate were designed using a computer software called Solidworks (2015 version, Dassault Systemes Solidworks Corp., USA). All the parameters, size, and distance are following the industry standard with reference of available literature (MacLeod et al., 2015). Both OTT and LCP systems have 5 screws and 3 screws orientation while the screw type was kept 
constant which using cortical screw. Further, the process was continued by fixing the bone plate to the fracture area using Mimic software. This implant placement was conducted with a reference from previous studies (Niemeyer et al., 2006; Zehnder et al., 2009). Figure 1 shows the plate placement on the ankle model.

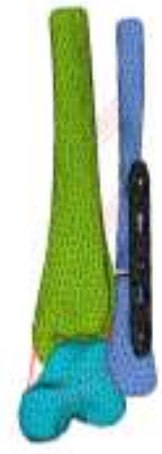

(b)

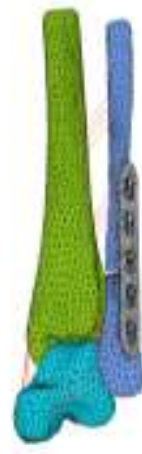

(b)

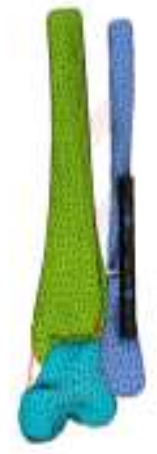

|at

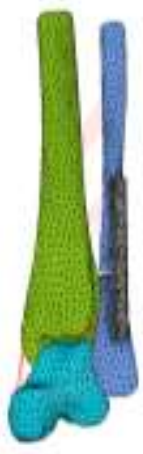

(ii)
Figure 1 Configuration of the plate and screws for; (a) 3 Screws (LCP) (b) 5 Screws (LCP) (c) 3 Screws (OTT) (d) 5 Screws (OTT).

\section{Screw placement}

The screw placement and number of screws used can be varied according to its application. The amount and placement of screw used need to be considered carefully to ensure even distribution of the load. While the addition to the number of screws will have no effect on the stability, reducing the number of screws used can improve the plate leverages, resulting in low load focus on each screw. To test this hypothesis, the present study was conducted by varying the placement and the number of screws. For this study, three and five screws were employed as suggested by Miller et al. (2007) where at least three screws should be used for each fragment. Figure 2 depicts the different orientations of screw for both designs.
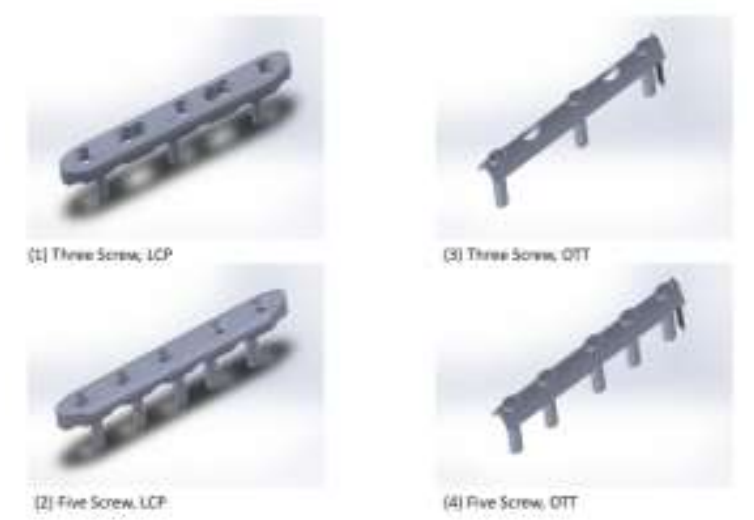

Figure 2 Plates of LCP and OTT with their number of screws.

\section{Finite element analysis}

For finite element analysis, axial loading has been chosen to be tested on the model due to standing condition. The force was positioned axially to the fibula and tibia. Note that the patient's body weight was $70 \mathrm{~kg}$, the load was distributed equally between two legs and was further distributed to tibia (93\%) and fibula (7\%) (Varsalona et al., 2006). As shown in Figure 3, the load to tibia and fibula are represented by yellow and red arrows, respectively. The load distributed between tibia and fibula was connected with link to imitate ligament. Besides, the model was also fixed at the bottom of talus surface to make sure the model was static. All friction coefficient values were set to 0.3 (Chen et al., 2004). The analysis was done using commercial finite element software Marc.Mentat (MSC.Software, Santa Ana, CA) with the equivalent von Mises stress (EVMS) and displacement of the model relative to the distal tibia.

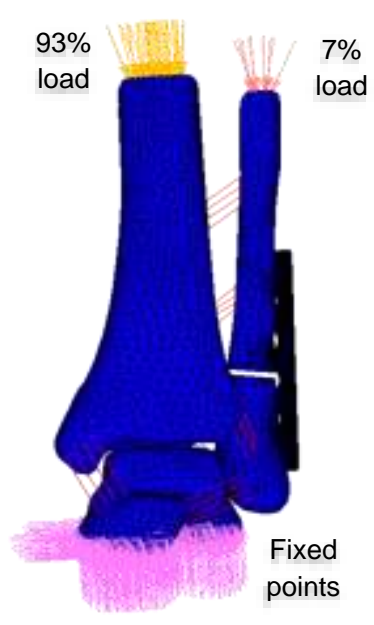

Figure 3 The finite element study of the model with the boundary conditions.

\section{RESULTS AND DISCUSSION}

\section{Stress Distribution}

The contour plots of von Mises stress (VMS) of the fibula and different configuration of screw for Locking Compression Plate (LCP) and One Third Tubular Plate (OTT) are shown in Figure 4 and 5, respectively. In general, the contour plots of all the configurations demonstrate that the bone had a high stress concentration at the pin-bone interface for stance phase.

Figure 7 and Figure 8 show maximum values of VMS at the pinbone interface and the highest VMS at bone plate. Generally, stresses at the bone plate was higher than fibula pin-bone interface. The graphs also include the value of ultimate strength of bone to measure the applicability of the model. For LCP, the highest VMS was observed for 3 screws $(183.7 \mathrm{MPa})$ at the fibula pin-bone interface, whereas for 5 screws was $70 \mathrm{MPa}$. Meanwhile, for OTT, the highest VMS was observed for 3 screws $(141.3 \mathrm{MPa})$ at the fibula pin-bone interface, whereas for 5 screws was $56 \mathrm{MPa}$.

Based on Figures 4 and 7, it is apparent that the value of stresses is still below than the ultimate strength of bone (193 MPa), indicating that all constructs of implant with different number of screws are safe to be used for patients without the possible risk of secondary fracture at the fibula bone. If the stresses values at the bone is higher than ultimate strength, therefore the medical surgeons need perform precaution steps to prevent secondary fracture from happening. For LCP, the highest VMS observed at the plate for 3 screws was $484 \mathrm{MPa}$, whereas for 5screw plate was $667 \mathrm{MPa}$. While, for OTT, the highest VMS at plate was observed for 3 screw was $300.5 \mathrm{MPa}$, whereas for 5-screw plate was $127.5 \mathrm{MPa}$.

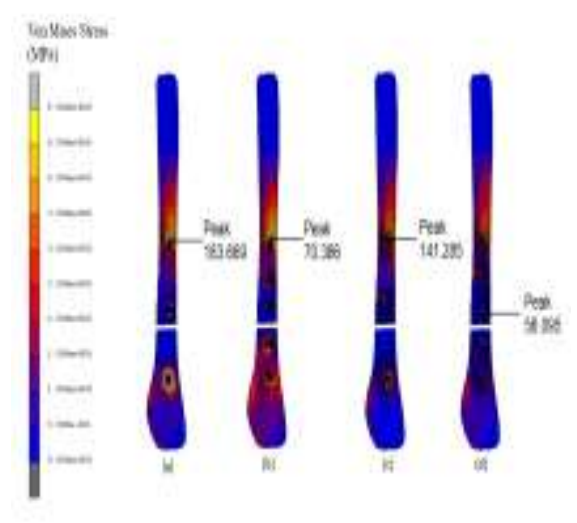

Figure 4 The contour plots of von Mises stress (VMS) of the fibula for different configuration of screw for Locking Compression Plate (LCP) (a) 3 screws (b) 5 screws and One Third Tubular (OTT) Plate (c) 3 screws (d) 5 screws. 


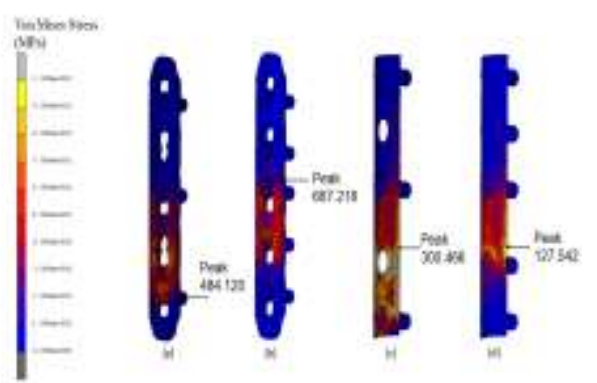

Figure 5 The contour plots of von Mises stress (VMS) at the plate and different configuration of screw for Locking Compression Plate (LCP) (a) 3 screw (b) 5 screws and One Third Tubular Plate (c) 3 screws (d) 5 screws.

\section{Displacement}

According to Denis et al. (2020), the stability of fracture can be defined by the number of deformation or displacement that occur at the fracture bone site. It should be noticed that a high displacement at the fracture bone can lead to unstable construct of treatment during full weight bearing exercise (Scheele at al., 2020).

Figure 6 shows the displacement plot of the fibula bone. Generally, the displacement value is below $4 \mathrm{~mm}$ for both LCP and OTT except for the OTT for 3 screws. When simulating the stance phase, LCP for 3 screws and 5 screws displacement are respectively slightly close at $3.59 \mathrm{~mm}$ and $3.51 \mathrm{~mm}$, while OTT for 3 screws and 5 screws displacement showing a big difference, at $5.65 \mathrm{~mm}$ and $3.72 \mathrm{~mm}$, respectively.

Conventional plating methods are based on the use of an adequate number of anchoring screws to press the plate against the bone with high compressive forces, creating a stable bone-implant connection. The revolutionary new aspect of the LCP is the combination of two completely different anchorage technologies in one implant. The importance of the reduction technique and minimally invasive plate insertion and fixation relates to ensuring that bone viability is undisturbed. Understanding of the biomechanical background of bridging plate osteosynthesis is essential if good clinical results are to be obtained (Niemeyer et al., 2006). This plate is also applied directly to the bone surface, so the force needs to be low to minimize the effect of shielding force. The reason shielding effect need to be minimised is to reduce the bone resorption process that will weaken the bone structure. Variables improving strength of fixation with conventional plates in normal bone has been extensively studied as well.

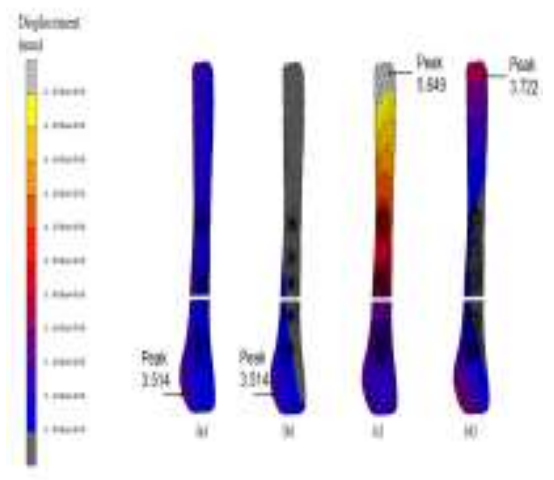

Figure 6 The displacement plot of the fibula bone for different configuration of screw for Locking Compression Plate (a) 3 screws (b) 5 screws and One Third Tubular Plate (c) 3 screws (d) 5 screws.
Factors shown to improve fixation strength include greater spacing of screws (Zehnder et al., 2009; DeCoster et al., 1990). In contrast to conventional plate osteosynthesis, when LCP are used, it is no longer possible to recommend a definite number of screws or cortices to be used in each fragment. While anchorage in the main fragments proximal and distal to the fracture zone remain important, it is much more important that the number of screws inserted is as small as is consistent with the provision of high plate leverage so that screw loading is kept low (Niemeyer et al., 2006). It has been reported that the gap size should be $1 \mathrm{~mm}$ and the number of screws of the plate should be varied (Miller et al., 2007; K. Stoffel et al., 2000).

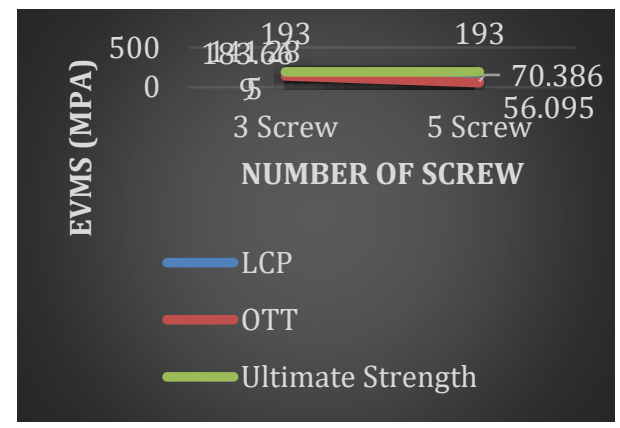

Figure 7 The graphs representing maximum values of VMS at the bone plate using two orientations of screw

In this study, some limitations and assumptions have been included due to the use of computer simulation to simulate the statics analysis instead of experimental works. First and foremost, the bones were assigned with isotropic and homogeneous properties, but the real conditions are anisotropic and inhomogeneous behaviour. However, it was found that the use of isotropic and homogenous properties for bone are more appropriate by considering the time consumed and computer resources (Frehill et al., 2019). Secondly, the use of VMS as a parameter to be discussed, not shear stress. Though the shear stress is good to be included, it is good to highlight in dynamics analysis and simulation. For this study, there was only statics analysis conducted where the use of VMS is more applicable as similar to previous finite element studies by others (Liu et al., 2019; Li et al., 2019). The next limitation was the choices of displacement where authors were discussing about the global and general displacement instead of displacement in $\mathrm{x}, \mathrm{y}$, and $\mathrm{z}$ directions ( $\mathrm{Li}$ et al., 2019). It is hoped that future studies can include all those three displacements to see which axis is more dominant.

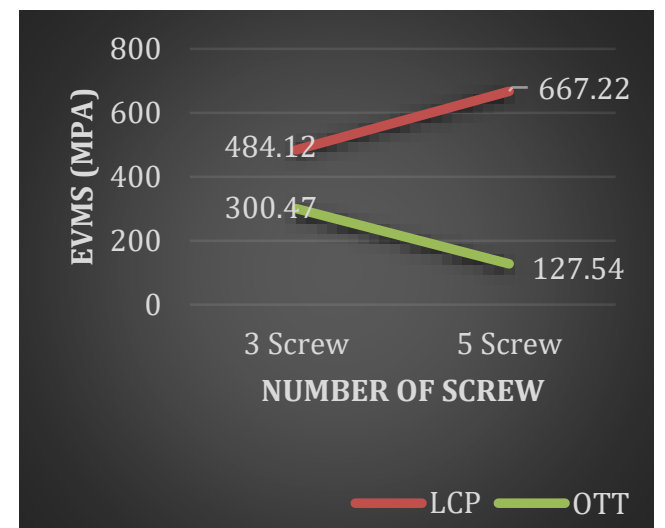

Figure 8 The graphs representing maximum values of VMS at the pinbone interface using two orientation of screw. 


\section{CONCLUSION}

On the basis of the results obtained, it can be noted that the usage of 3 screws causing a lower VMS at plate compare to 5 screws for LCP construct. In terms of displacement, the value is quite close for both 5 screws and 3 screws for LCP but the difference is clear for OTT model. Therefore, this study was successfully conducted to compare the stability of stress distribution of LCP and OTT for providing related information to medical surgeons of their choices in treating patient.

\section{ACKNOWLEDGEMENT}

This work was financially supported by the Universiti Teknologi Malaysia (UTM) under the Research University Grant (Tier 2: 15J84) and Ministry of Education Malaysia (Fundamental Research Grant Scheme: 5F135). We would like to thank UTM for providing us with good facilities to conduct this research.

\section{REFERENCES}

Shibuya, N., M. L. Davis, D. C. Jupiter. Epidemiology of foot and ankle fractures in the United States: An analysis of the National Trauma Data Bank (2007 to 2011). J. Foot Ankle Surg., 2014. 53(5): p. 606-608.

Labronici, P. J., R. E. Pires, M. V. Franco, R. Freitas, G. C. Araujo et al. Medial malleolar fractures: An anatomic survey determining the ideal screw length. Ann. Med. Health Sci. Res., 2016. 6(5): p. 308-310.

Raja Izaham, R. M., M. R. A. Kadir, A. H. A. Rashid, M. G. Hossain, T. Kamarul. Finite element analysis of Puddu and Tomofix plate fixation for open wedge high tibial osteotomy. Injury, 2012. 43(6): p. 898-902.

Chen W. P., Tai, C. L., Chih, C. H., Hsieh, P. H., Leou, M. C., Lee, M. S. Selection of fixation devices in proximal femur rotational osteotomy: clinical complications and finite element analysis. Clin. Biomech., 2004. 19: p.255-262.

MacLeod, A. R., A. H. Simpson, P. Pankaj. Reasons why dynamic compression plates are inferior to locking plates in osteoporotic bone: A finite element explanation. Comput. Methods Biomech. Biomed. Engin., 2015. 18(16): p. 1818-1825.

Miller, D. L., T. Goswami. A review of locking compression plate biomechanics and their advantages as internal fixators in fracture healing. Clin. Biomech (Bristol, Avon), 2007. 22(10): p. 1049-1062.

Varsalona, R., G. T. Liu. Distal tibial metaphyseal fractures: The role of fibular fixation. Strategies in Trauma and Limb Reconstruction, 2006. 1(1): p. 4250 .

P. Niemeyer, N. P., Südkamp. Principles and clinical application of the locking compression plate (LCP), Acta Chirurgiae Orthopaedicae Et Traumatologiae Čechosl., 73, 2006, p. 221-228.

Zehnder, S., J. G. Bledsoe, A. Puryear. The effects of screw orientation in severely osteoporotic bone: A comparison with locked plating. Clin. Biomech (Bristol, Avon), 2009. 24(7): p. 589-594.

DeCoster et al., Optimizing Bone Screw Pullout Force. Journal of Orthopaedic Trauma, 1990. 4(2): p. 169-174.

K. Stoffel, K. Klaue, S. M. Perren. Functional load of plates in fracture fixation in vivo and its correlate in bone healing. Injury, 31 (2000) S-B37-SB50.

Denis, D., D. M. Boulch, Y. Suchier, T. B. Mamadou, J. D., Metaizeau. Biomechanical and clinical comparative study of the new elastic stable intramedullary nailing "MJ-FLEX Orthofix", J. Pediatr. Prthop., 2020. 40(3), p. 149-155.

Scheele, C. B., M. F. Pietschmann, C. Schröder, F. Lenze, T. M. Grupp, P. E. Müller. Effect of bone density and cement morphology on biomechanical stability of tibial unicompartmental knee arthroplasty. Knee, 2020. Feb 2, S0968-0160(20)30004-1.

Frehill, B., A. D. Crocombe. Finite element assessment of metaphyseal sleeves in total knee arthroplasty. J. of Ortho, 2019. 18(19), p. 1-8.

Liu X., F. Pang, Y. Li, H. Jia, X. Cui, Y.Yue, X. Yang, Q. Yang. Effects of differrent positions and angles of implants in maxillary edentulous jaw on surrounding bone stress under dynamic loading: A three-dimensional finite element analysis. Comp. and Math. Meth. in Med., 2019. 1-9.

Li, Y. R., Y. H. Gao, C. Yang, L. Ding, X. Zhang, H. Chen, J. Liu, X. Qi. Finiteelement analysis of the proximal tibial sclerotic bone and different alignment in total knee arthroplasty. BMC Muscu. Dis., 2019. 20(617), p.19. 